\title{
Forecasting Cross-Section of Stock Returns with Realised Moments
}

\author{
Milan Fičura*
}

\begin{abstract}
:
The study tests whether realised moments of stock returns (mean, variance, skewness and kurtosis) computed from daily returns over the last month, quarter and year can predict the 1-month cross-sectional stock returns of 40 US-traded liquid stocks in the period 1986-2019. The performed univariate regression analysis confirmed a statistically significant positive effect between all the realised moments, computed over the last quarter and year, and the future 1-month crosssectional stock returns, while the 1-month realised moments proved to be mostly insignificant. Multivariate analysis, performed with Elastic Net Regression, has confirmed that investment strategies utilising information from realised moments were able to significantly outperform a random investment in the out-sample period 2004-2019.
\end{abstract}

Key words: Cross-Section of Stock Returns; Realised variance; Realised Skewness; Realised Kurtosis; Momentum Effect

JEL classification: G11, G12, G17

\section{Introduction}

The goal of the presented study is to assess whether realised moments of past stock price returns can be used to predict cross-section of stock returns in the 1month horizon and whether a portfolio constructed by using information from realised moments is able to statistically significantly outperform a random investment.

The momentum effect of the realised mean on future stock returns is a well-known anomaly in finance. The statistically significant effect of cross-sectional momentum was studied in the works of Jegadeesh and Titman (1993) and

\footnotetext{
* Milan Fičura; University of Economics, Prague, Faculty of Finance and Accounting, Department of Banking and Finance, W. Churchill Sq. 4, 130 67, Prague 3, Czech Republic, <milan.ficura@vse.cz>.

The article is processed as an output of the research project Innovation Approaches to Credit Risk Management registered by the Czech Science Foundation (GACR) under the registration number GAČR 18-05244S, and the research project Advanced methods of risk management with the use of artificial intelligence VŠE IGS F1/59/2017.
} 
Jegadeesh and Titman (2002), while the effect of single time-series momentum is studied in Moskowitz (2012) or Bird et al. (2016).

The effect of realised variance on future stock returns has been widely discussed in literature. While the positive effect of the systematic part of the variance on future returns is commonly acknowledged, the effect of the idiosyncratic variance is less clear, with Ang et al. (2006) reporting a negative effect, while Xu and Malkiel (2004) identifying a positive effect.

In recent years, multiple studies have reported statistically significant effects of realised skewness and kurtosis on future returns. Amaya et al. (2015) identified a significant negative effect of weekly realised skewness, computed from highfrequency data, on the cross-sectional performance of stocks over the next week, with a weaker positive effect identified also for the realised kurtosis. Liu et al. (2014) present a noise-robust high-frequency estimator of realised skewness, confirm its predictive power with respect to the 1-month US stock returns, and link it to the time-variability of the market risk premium. Shen et al. (2018), on the other hand, do not find a statistically significant effect of realised skewness on 1day returns of individual stocks.

In the presented study, we will evaluate the effect of the realised mean, variance, skewness and kurtosis, computed from daily returns over a monthly, quarterly and yearly aggregation period, on the expected cross-sectional returns of stocks in the following month. The analysis is performed on the portfolio of 40 highly liquid US-traded stocks that either were, or currently are, a part of the DJIA index.

In addition to the univariate analysis, the Elastic Net Regression model (Zhou and Hastie, 2005) is estimated with all of the realised moments as potential predictors, and the performance of portfolio investing according to the predictions of the model is compared with equal-weight and random investments.

The rest of the study is organised as follows. In Section 2 we define the realised moments used in the rest of the study. Section 3 explains the computation of crosssectional normalised returns and moments. Section 4 explains the Elastic Net Regression model. In Section 5 the dataset is discussed, while Sections 6 and 7 contain the univariate and multivariate analyses, respectively. The conclusion contains a summary of the main findings

\section{Realised Moments}

Realised moments represent estimates of the return moments on some lower frequency (in our case monthly, quarterly and yearly), computed from returns on a higher frequency (in our case daily). 
Let us define as $t$ the end time of the aggregation period (month, quarter or year), comprising of $N$ trading days indexed $i=1, \ldots, N$.

The logarithmic return during day $i$ can be defined as:

$$
r_{i}=p_{i}-p_{i-1}
$$

where $p_{i}$ denotes the logarithm of the closing price on day $i$.

The realised mean at time $t$ over the aggregation period of size $N$, ending at $t$, is equal to the total return over $N$, computed as a sum of the daily returns:

$$
\operatorname{RMean}_{t, N}=\sum_{i=1}^{N} r_{t, i},
$$

while the realised variance is:

$$
\operatorname{RVar}_{t, N}=\sum_{i=1}^{N}\left(r_{t, i}-\mu_{t, N}\right)^{2}
$$

where $\mu_{t, N}$ denotes the daily average return over the period $N$ ending at $t$ :

$$
\mu_{t, N}=\frac{1}{N} \operatorname{RMean}_{t, N}=\frac{1}{N} \sum_{i=1}^{N} r_{t, i} .
$$

In addition to the realised variance, we will also use the realised volatility:

$$
R \operatorname{Vol}_{t, N}=\sqrt{\operatorname{RVar}_{t, N}}
$$

and the volatility adjusted mean:

$$
\operatorname{RAdjMean}_{t, N}=\frac{\operatorname{RMean}_{t, N}}{\operatorname{RVol}_{t, N}} .
$$

The realised skewness is further defined as:

$$
\operatorname{RSkew}_{t, N}=\frac{\sqrt{N} \sum_{i=1}^{N}\left(r_{t, i}-\mu_{t, N}\right)^{3}}{\operatorname{RVar}_{t, N}^{3 / 2}}
$$

and the realised kurtosis as:

$$
\operatorname{RKurt}_{t, N}=\frac{N \sum_{i=1}^{N}\left(r_{t, i}-\mu_{t, N}\right)^{4}}{\operatorname{RVar}_{t, N}^{2}}
$$

Often-used approximation in financial econometrics assumes that $\mu_{t, N}=0$. In accordance with Amaya et al. (2015) we therefore also define the zero-mean versions of the moments above.

Zero-mean realised variance is:

$$
\operatorname{RVar}_{\mu=0, t, N}=\sum_{i=1}^{N} r_{t, i}^{2},
$$

zero-mean realised volatility: 
Fičura, M.: Forecasting Cross-Section of Stock Returns with Realized Moments.

$$
R V o l_{\mu=0, t, N}=\sqrt{\operatorname{RVar}_{\mu=0, t, N}},
$$

zero-mean realised skewness:

$$
\operatorname{RSkew}_{\mu=0, t, N}=\frac{\sqrt{N} \sum_{i=1}^{N} r_{t, i}^{3}}{R V a r_{t, N}^{3 / 2}},
$$

and zero-mean realised kurtosis:

$$
\operatorname{RKurt}_{\mu=0, t, N}=\frac{N \sum_{i=1}^{N} r_{t, i}^{4}}{R V a r_{t, N}^{2}}
$$

Within the empirical study covered in the following sections, the realised moments are computed for the monthly $(N=22)$, quarterly $(N=66)$ and yearly $(N=264)$ aggregation period and used as predictors of the future 1-month cross-sectional stock returns.

\section{Cross-Sectional Moments and Returns}

As the goal of the study is to predict relative cross-sectional returns of stocks regardless of the movements of the overall market, the moments and returns need to be normalised over the whole portfolio consisting of $M$ stocks.

Let us denote as $r_{t, i, j}$ the return of the stock $j$, for $j=1, \ldots, M$, in the day $i$, for $i=$ $1, \ldots, N$, in the aggregation period ending at $t$, consisting of $N$ days. Unlike the moments, returns will always be predicted for a monthly horizon, i.e. for $N=22$, and we thus omit $N$ in the notation.

The total return of the stock $j$ over the period $t$ can be computed as:

$$
r_{t, j}=\sum_{i=1}^{N} r_{t, i, j}
$$

The average stock return in the period $t$ is computed as:

$$
\mu_{r, t}=\frac{1}{M} \sum_{j=1}^{M} r_{t, j}
$$

and the volatility of the stock returns in the period $t$ is computed as:

$$
\sigma_{r, t}=\sqrt{\sum_{j=1}^{M}\left(r_{t, j}-\mu_{r, t}\right)^{2}}
$$

The normalised relative return of the stock $j$ in the period $t$ is then:

$$
r_{t, j}^{*}=\frac{r_{t, j}-\mu_{r, t}}{\sigma_{r, t}}
$$

In the same way, the predictor variables (i.e. realised moments defined in the previous section) can be normalised as well. 
We define $X_{t, j, N}$ as a given predictor computed for the stock $j$ at the aggregation period $t$, consisting of $N$ trading days.

The mean value of the predictor value can be defined as:

$$
\mu_{X, t}=\frac{1}{M} \sum_{j=1}^{M} X_{t, j, N},
$$

and its volatility as:

$$
\sigma_{X, t}=\sqrt{\sum_{j=1}^{M}\left(X_{t, j}-\mu_{X, t}\right)^{2}} .
$$

The normalised value of the predictor value for the stock $j$ at the period $t$ is then:

$$
X_{t, j, N}^{*}=\frac{X_{t, j}-\mu_{X, t}}{\sigma_{X, t}}
$$

\section{Elastic Net Regression}

The Elastic Net Regression (Zhou and Hastie, 2005) is a linear regression-based method that combines the Ridge regression penalisation on the $L_{2}$ norm of the parameter vector to reduce overfitting, and the LASSO regression penalisation on the $L_{1}$ norm of the parameter vector to perform variable selection.

The parameter estimates $\beta$ are computed with the following optimisation:

$$
\hat{\beta} \equiv \min _{\beta}\left(\|y-X \beta\|^{2}+\lambda_{2}\|\beta\|^{2}+\lambda_{1}\|\beta\|_{1}\right),
$$

where $y$ denotes the vector of targets, $X$ is the matrix of predictors, and $\beta$ is the vector of regression parameters.

Meta-parameters $\lambda_{2}$ and $\lambda_{1}$ express the strength of the penalisation on the $L_{2}$ and the $L_{1}$ norm of the parameter vector, respectively. Within the performed study they are determined with 10 -fold cross-validation and run on the in-sample period of the data with the goal of maximising the in-sample mean squared error of the predictions.

\section{Data}

The utilised dataset consists of daily prices of stocks that either were, or currently are, a part of the DJIA stock index and that were tradable during the whole period from the start of 1986 until the end of January 2019. Former DJIA components were included into the dataset in order to partially alleviate the possible survivorship bias of the sample (the stocks that remained in DJIA are the relatively better performing ones, which might favour contrarian investment strategies). In cases where the company that used to be in DJIA was subject to a merger, the 
Fičura, M.: Forecasting Cross-Section of Stock Returns with Realized Moments.

stock price of the new merged company was used instead. As a result of the applied selection rules and data constrains, we were left with 40 stocks to be used in the analysis. The list of the stocks can be found in Appendix 1. Data were provided by Yahoo Finance.

Target returns were computed on a monthly basis at the end of each month. The predictors were computed from the daily returns with aggregation periods of 1-month, 3-months and 12-months, proceeding the target month.

\section{Univariate Analysis}

Tab. 1 Univariate regressions between normalised 1-month stock returns and normalised realised moments (data from 1986-2019)

\begin{tabular}{lrrr}
\hline Predictor & Slope & t-stat & p-value \\
\hline RMean $_{t, 22}^{*}$ & -0.0097 & -1.2003 & 0.2300 \\
RVol $_{t, 22}^{*}$ & -0.0025 & -0.3086 & 0.7576 \\
RSkew $_{t, 22}^{*}$ & 0.0117 & 1.4533 & 0.1461 \\
RKurt $_{t, 22}^{*}$ & -0.0055 & -0.6842 & 0.4939 \\
RAdjMean $_{t, 22}^{*}$ & -0.0246 & -3.0524 & 0.0023 \\
RMean $_{t, 66}^{*}$ & 0.0364 & 4.5270 & $6.03 \mathrm{E}-06$ \\
RVol $_{t, 66}^{*}$ & 0.0336 & 4.1710 & $3.05 \mathrm{E}-05$ \\
RSkew $_{t, 66}^{*}$ & 0.0426 & 5.3031 & $1.15 \mathrm{E}-07$ \\
RKurt $_{t, 66}^{*}$ & 0.0513 & 6.3827 & $1.79 \mathrm{E}-10$ \\
RAdjMean $_{t, 66}^{*}$ & 0.0139 & 1.7276 & 0.0841 \\
RMean $_{t, 264}^{*}$ & 0.0683 & 8.5044 & 0 \\
RVol $_{t, 264}^{*}$ & 0.0350 & 4.3516 & $1.36 \mathrm{E}-05$ \\
RSkew $_{t, 264}^{*}$ & 0.0573 & 7.1334 & $1.02 \mathrm{E}-12$ \\
RKurt $_{t, 264}^{*}$ & 0.0313 & 3.8894 & 0.0001 \\
RAdjMean $_{t, 264}^{*}$ & 0.0491 & 6.1069 & $1.04 \mathrm{E}-09$ \\
RVol $_{\mu=0, t, 22}^{*}$ & -0.0032 & -0.3996 & 0.6895 \\
RSkew $_{\mu=0, t, 22}^{*}$ & -0.0049 & -0.6083 & 0.5430 \\
RKurt $_{\mu=0, t, 22}^{*}$ & -0.0057 & -0.7139 & 0.4753 \\
RAdjMean $_{\mu=0, t, 22}^{*}$ & -0.0242 & -3.0068 & 0.0026 \\
RVol $_{\mu=0, t, 66}^{*}$ & 0.0337 & 4.1936 & $2.76 \mathrm{E}-05$ \\
RSkew $_{\mu=0, t, 66}^{*}$ & 0.0394 & 4.9005 & $9.66 \mathrm{E}-07$ \\
RKurt $_{\mu=0, t, 66}^{*}$ & 0.0523 & 6.5121 & $7.64 \mathrm{E}-11$ \\
RAdjMean $_{\mu=0, t, 66}^{*}$ & 0.0141 & 1.7476 & 0.0805 \\
RVol $_{\mu=0, t, 264}^{*}$ & 0.0355 & 4.4130 & $1.03 \mathrm{E}-05$
\end{tabular}




\begin{tabular}{llll} 
RSkew $_{\mu=0, t, 264}^{*}$ & 0.0586 & 7.2957 & $3.12 \mathrm{E}-13$ \\
RKurt $_{\mu=0, t, 264}^{*}$ & 0.0323 & 4.0211 & $5.82 \mathrm{E}-05$ \\
RAdjMean $_{\mu=0, t, 264}^{*}$ & 0.0491 & 6.1107 & $1.02 \mathrm{E}-09$ \\
\hline
\end{tabular}

Source: Authorial computation based on data from Yahoo Finance.

In the first step, a series of univariate regressions was computed between each of the predictors $X_{t, j, N}^{*}$ and the normalised returns in the following month $r_{t+1, j}^{*}$. The estimation was performed over the period 1986-2019 for the portfolio of 40 stocks as defined in Section 5. The results of regressions are shown in Table 1.

It is apparent from Table 1 that most of the realised moments had a statistically significant impact on the relative returns in the 1-month horizon.

The positive momentum effect of the realised mean is clearly apparent for the 3month and the 12-month aggregation period, while for the 1-month aggregation period, the realised mean has an opposite (i.e. negative) effect on future returns.

The effect of realised volatility on future 1-month returns is positive and significant for the 3-month and 12-month aggregation period. The same is true also for the realised skewness and the realised kurtosis. The type of computation (i.e. whether we apply the assumption that $\mu=0$ or not) does not seem to have a dramatic effect on the results of the analysis.

\section{Portfolio Returns}

In order to test whether the identified relationships can be used to achieve aboveaverage portfolio returns, the dataset was divided into the in-sample period (19862003) and the out-sample period (2004-2019), and a multi-variate regression model was estimated on the in-sample period with the Elastic Net Regression model. To cope with multicollinearity of some of the predictors, in addition to the raw explanatory variables the model was alternatively applied also to their principal components computed with Principal Component Analysis.

In turn, the following investment strategies were constructed and tested:

1. Long Only - at the end of each month we enter into long position in 8 stocks (20\% of the total number) for which the model predicts the highest normalised return.

2. Long/Short - at the end of each month we enter into long position in 8 stocks for which the model predicts the highest normalised return, and into short position in 8 stocks for which it predicts the lowest normalised return. 
As the model gives us only information about which stocks will perform better than others and not what their returns will be, we cannot construct a Short Only strategy.

For the initial evaluation of the performance, the transaction costs (spreads) are omitted and the short positions are assumed to neither bear any additional costs, nor generate proceeds that could be re-invested.

As a primary benchmark strategy, an equal investment in all of the stocks at the start of the out-sample period is considered.

In order to evaluate the significance of the results, 1,000 random strategies (alternatively based on the Long Only or the Long/Short setting) were generated, calculating the score at the end of each month randomly and allocating their investments according to the rules mentioned above. Confidence intervals (95\%) were then computed for the cumulative profits.

Figures 1 and 2 show the cumulative returns of the Long Only and the Long Short strategy under the setting where the scores were computed with the multivariate Elastic Net Regression applied to the realised moments directly. Figures 3 and 4 show the results for the case where the Principal Component Analysis (PCA) was utilised and the Elastic Net Regression was applied to the principal components.

\section{Fig. 1 Out-Sample cumulative return of the Long Only strategy}

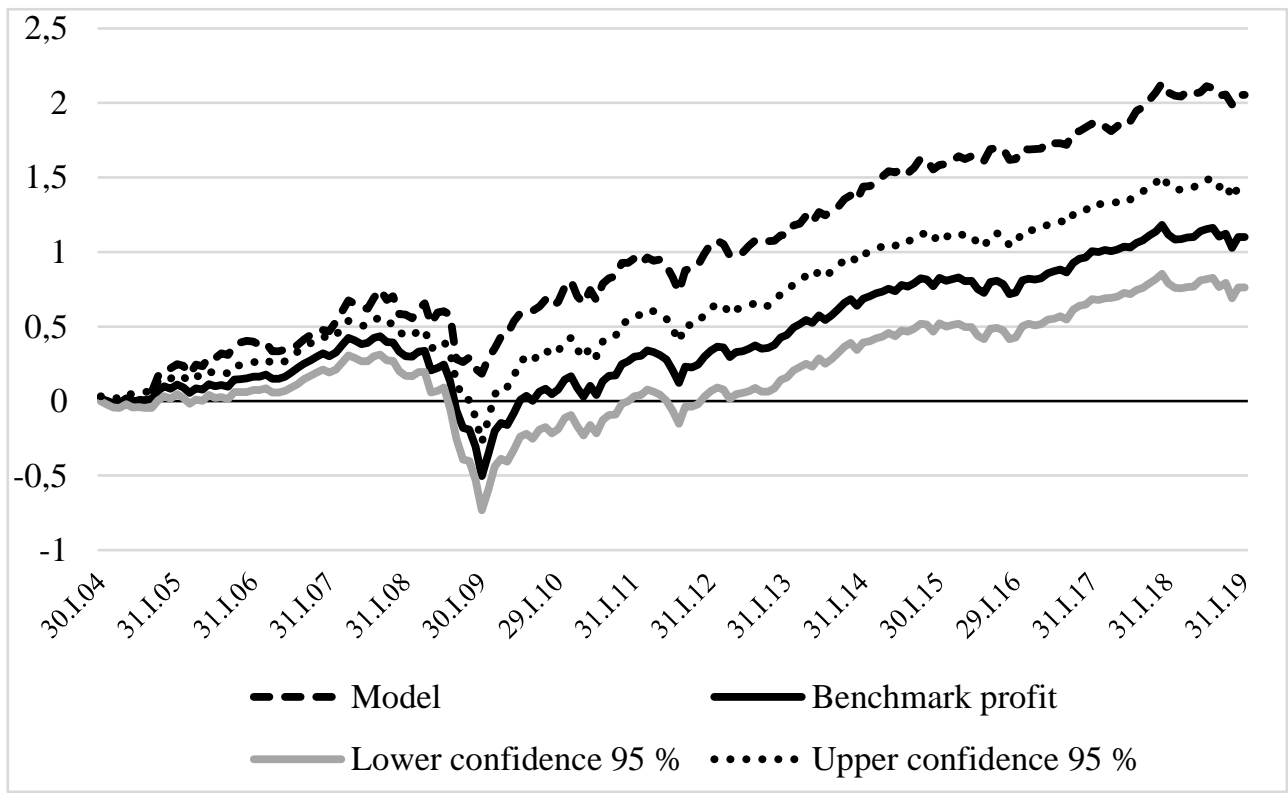


Source: Authorial computation based on data from Yahoo Finance.

Fig. 2 Out-Sample cumulative return of the Long/Short strategy

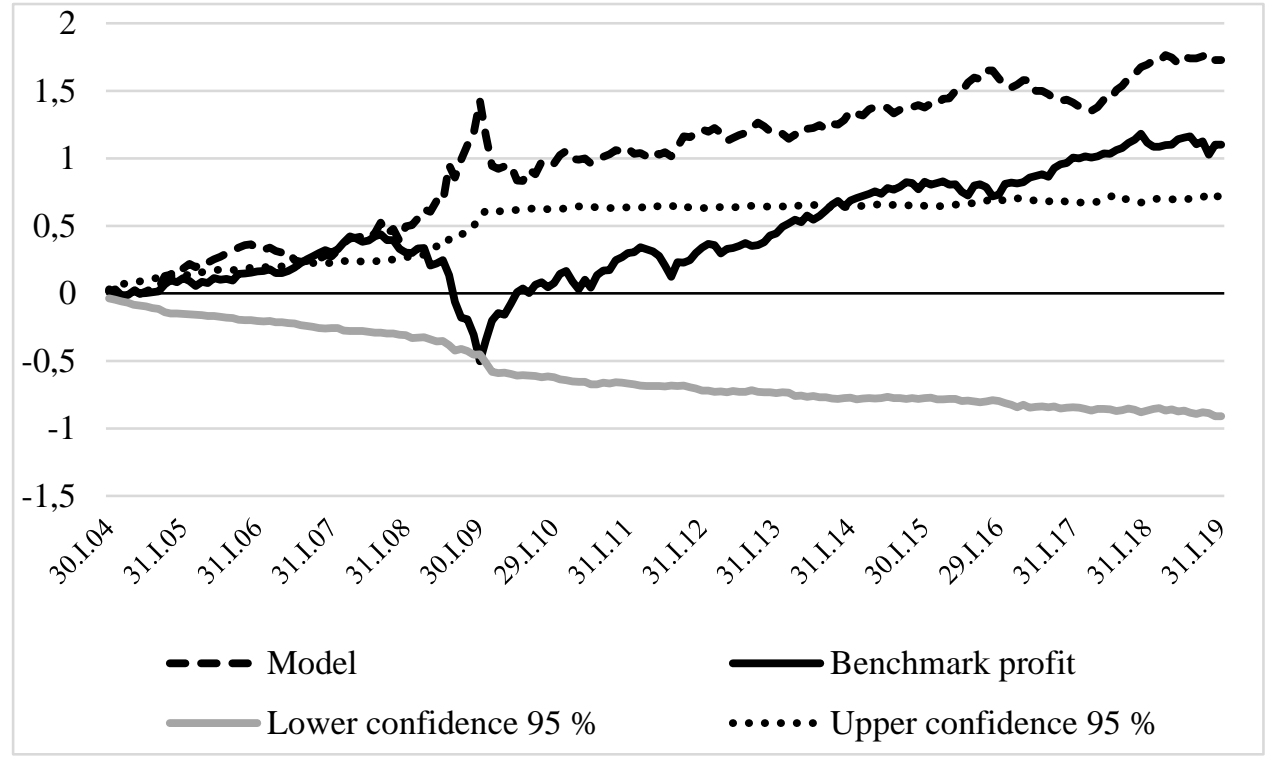

Source: Authorial computation based on data from Yahoo Finance.

Fig. 3 Out-Sample cumulative return of the Long Only strategy (PCA)

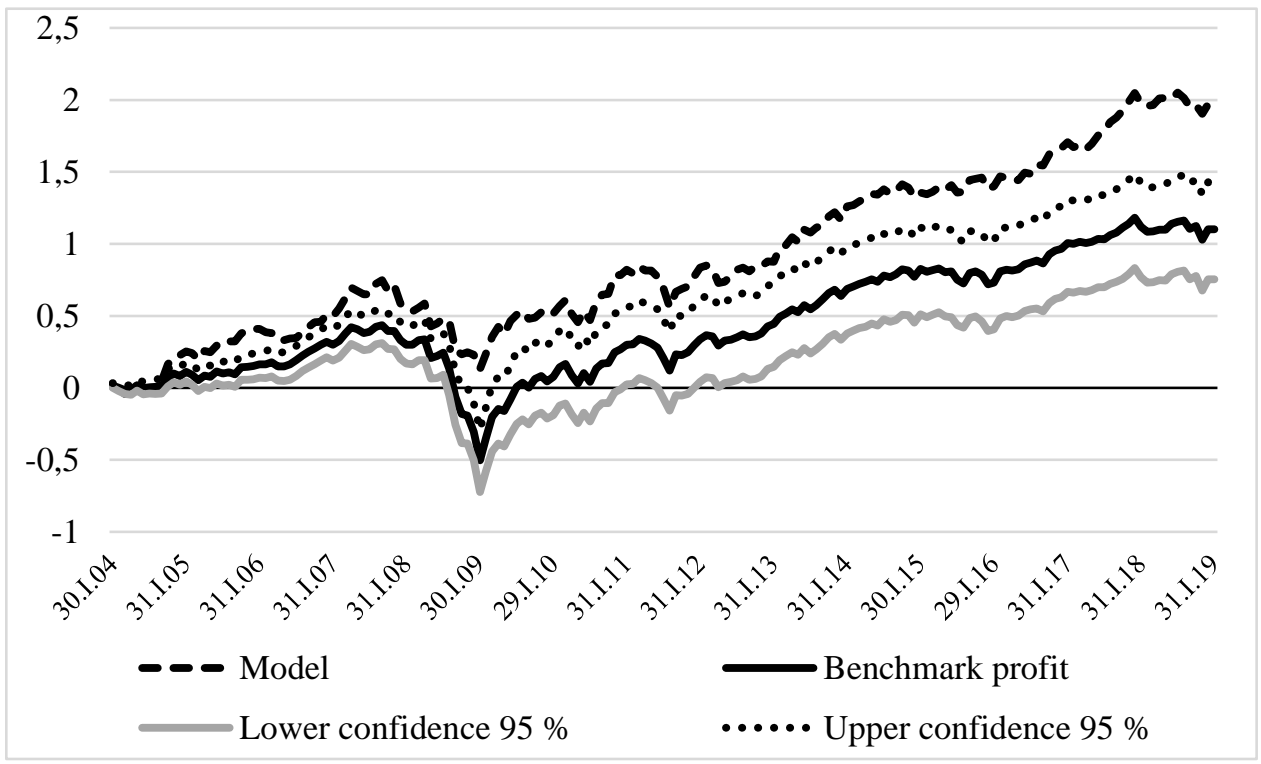


Source: Authorial computation based on data from Yahoo Finance.

Fig. 4 Out-Sample cumulative return of the Long/Short strategy (PCA)

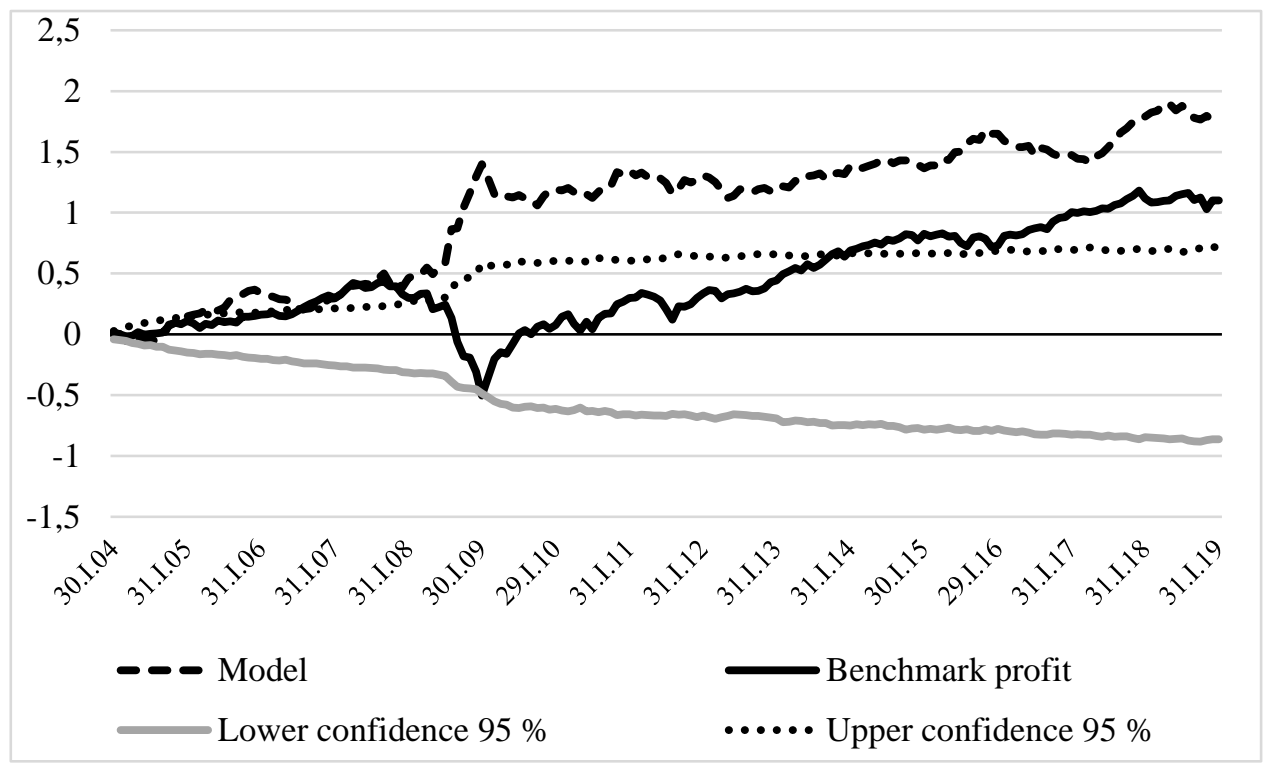

Source: Authorial computation based on data from Yahoo Finance.

The tested strategies outperformed the Benchmark as well as the upper 95\% confidence bound of a random investment. The Long Only strategies exhibited a drop during 2008-2009, but it was lower than for the Benchmark and they managed to systematically outperform the Benchmark over the following years. The Long/Short strategies profited strongly in 2008-2009, with a slightly worse than Benchmark performance in the following years.

Table 2 shows the annual returns, volatility and Sharpe ratios for all the strategies. Table 3 shows the statistics in the case when $0.2 \%$ spread is assumed for entering into positions. The spread corresponds to the results in Jones (2002) for the DJIA index in year 2000, providing a conservative estimate for the period 2004-2019.

Tab. 2 Performance metrics of the tested strategies (no spread)

\begin{tabular}{lrrr}
\hline Model type & Return p.a. & StDev p.a. & Sharpe p.a. \\
\hline Benchmark & $7.19 \%$ & $16.47 \%$ & 0.4364 \\
Long Only & $12.47 \%$ & $17.43 \%$ & 0.7157 \\
Long/Short & $8.04 \%$ & $19.65 \%$ & 0.4090 \\
Long Only (PCA) & $12.55 \%$ & $17.69 \%$ & 0.7092 \\
Long/Short (PCA) & $11.08 \%$ & $16.46 \%$ & 0.6729 \\
\hline
\end{tabular}


Source: Authorial computation based on data from Yahoo Finance.

Tab. 3 Performance metrics of the tested strategies $(0.2 \%$ spread $)$

\begin{tabular}{lrrr}
\hline Model type & Return p.a. & StDev p.a. & Sharpe p.a. \\
\hline Benchmark & $7.19 \%$ & $16.47 \%$ & 0.4364 \\
Long Only & $10.09 \%$ & $17.43 \%$ & 0.5788 \\
Long/Short & $5.65 \%$ & $19.65 \%$ & 0.2875 \\
Long Only (PCA) & $10.16 \%$ & $17.69 \%$ & 0.5743 \\
Long/Short (PCA) & $8.69 \%$ & $16.46 \%$ & 0.5279 \\
\hline
\end{tabular}

Source: Authorial computation based on data from Yahoo Finance.

It is apparent that even with a conservative assumption for the spread sizes, the Long Only strategies and the Long/Short (PCA) strategy outperformed the Benchmark.

\section{Conclusion}

The study analysed the relationship between realised moments (mean, variance, skewness and kurtosis) computed over the 1-month, 3-month and 12-month period, and the future 1-month cross-sectional stock returns of a portfolio of 40 US-traded stocks in the period from 1986 to 2019. Results of the univariate analysis confirmed the existence of a strong positive relationship between the realised moments computed over the period of 3 or 12 months, and the stock returns in the following month. The 1-month moments were, on the other hand, mostly insignificant with the exception of the volatility-adjusted mean which had a negative effect on future returns. Long Only and Long/Short strategies were proposed, utilising the Elastic Net Regression model estimated between the realised moments and the cross-sectional stock returns in the period 1986-2003. The returns of these strategies significantly outperformed a random investment in the out-sample period (2004-2019). The results thus confirm that the realised moments contain useful information for stock returns prediction and portfolio investment.

\section{References}

Amaya, D., Christoffersen, P., Jacobs, K., Vasquez A., 2015. Does realized skewness predict the cross-section of equity returns? Journal of Financial Economics, 118(1), 135-167. DOI: 10.2139/ssrn.1785736.

Ang, A., Hodrick, R. J., Xing, Y., Zhang, X., 2004. The Cross-Section of Volatility and Expected Returns. NBER Working Paper No. 10852, DOI: 10.3386/w10852. 
Bird, R., Gao, Y., Yeung, D., 2016. Time-series and cross-sectional momentum strategies under alternative implementation strategies. Australian Journal of Management, 42(2), 230-251. DOI: 10.1177/0312896215619965.

Jegadeesh, N., Titman, S., 1993. Returns to Buying Winners and Selling Losers: Implications for Stock Market Efficiency. The Journal of Finance, 48(1), 65-91. DOI: $10.2307 / 2328882$.

Jegadeesh, N., Titman, S., 2001. Profitability of Momentum Strategies: An Evaluation of Alternative Explanations. The Journal of Finance, 56(2), 699720. DOI: 10.1111/0022-1082.00342.

Jones, C. M., 2002, A Century of Stock Market Liquidity and Trading Costs. SSRN Electronic Journal. DOI: 10.2139/ssrn.313681.

Liu, Z., Wang, K., Liu, J., 2014. Realized Skewness at High Frequency and the Link to a Conditional Market Premium. SSRN Electronic Journal. Available from: <ssrn.com/abstract=2224216>. [28 August 2019].

Moskowitz, T. J., Ooi, Y. H., Pedersen, L. H., 2012. Time Series Momentum. Journal of Financial Economics. 104(2), 228-250. Available from: <docs.lhpedersen.com/ TimeSeriesMomentum.pdf>. [28 August 2019].

Shen, K., Yao, J., Li, W. K., 2018. On the Surprising Explanatory Power of Higher Realized Moments in Practice. Statistics and Its Interface. 11(1), 153168. DOI: 10.4310/sii.2018.v11.n1.a13.

Xu, Y., Malkiel, G. M., 2004. Idiosyncratic Risk and Security Returns. SSRN Electronic Journal. DOI: 10.2139/ssrn.255303.

Zhou, H., Hastie, T., 2005. Regularization and variable selection via the Elastic Net. Journal of the Royal Statistical Society, Series B (Statistical Methodology). 67(5). DOI: 10.1111/j.1467-9868.2005.00527.x. 
European Financial and Accounting Journal, 2019, vol.14, no. 2, pp. 71-84.

\section{Appendix 1 List of stocks in the portfolio}

\begin{tabular}{|c|c|c|c|}
\hline Company & Exchange & Symbol & Industry \\
\hline $3 \mathrm{M}$ & NYSE & MMM & Conglomerate \\
\hline American International Group & NYSE & AIG & Financial services and Insurance \\
\hline Alcoa & NYSE & AA & Metals \\
\hline Altria Group & NYSE & MO & Tobacco \\
\hline American Express & NYSE & AXP & Consumer finance \\
\hline Apple & NASDAQ & AAPL & Consumer electronics \\
\hline ArcelorMittal & NYSE & MT & Steel \\
\hline AT\&T & NYSE & $\mathrm{T}$ & Telecommunication \\
\hline Bank of America & NYSE & $\mathrm{BAC}$ & Investment banking \\
\hline Boeing & NYSE & BA & Aerospace anddefense \\
\hline Caterpillar & NYSE & CAT & Construction andmining equipment \\
\hline Chevron & NYSE & CVX & Oil \& gas \\
\hline CBS Corporation & NYSE & CBS & Mass media \\
\hline Cisco Systems & NASDAQ & $\mathrm{CSCO}$ & Computer networking \\
\hline Citigroup & NYSE & $\mathrm{C}$ & Banking \\
\hline Coca-Cola & NYSE & $\mathrm{KO}$ & Beverages \\
\hline Du Pont & NYSE & DWDP & Chemical industry \\
\hline ExxonMobil & NYSE & $\mathrm{XOM}$ & Oil \& gas \\
\hline Foot Locker & NYSE & FL & Clothing \\
\hline General Electric & NYSE & GE & Conglomerate \\
\hline General Motors & NYSE & GM & Automotive \\
\hline Goldman Sachs & NYSE & GS & Banking, Financial services \\
\hline Goodyear Tire \& Rubber & NASDAQ & GT & Manufacturing \\
\hline Hewlett Packard & NYSE & HPQ & Computer hardware \\
\hline Honeywell & NYSE & $\mathrm{HON}$ & Conglomerate \\
\hline Home Depot & NYSE & HD & Home improvementretailer \\
\hline IBM & NYSE & IBM & Computers and technology \\
\hline Intel & NASDAQ & INTC & Semiconductors \\
\hline International Paper & NYSE & IP & Pulp and Paper \\
\hline Johnson \& Johnson & NYSE & JNJ & Pharmaceuticals \\
\hline JPMorgan Chase & NYSE & JPM & Banking \\
\hline Kraft Heinz & NASDAQ & $\mathrm{KHC}$ & Food \& Beverages \\
\hline Kodak & NYSE & KODK & Imaging technology \\
\hline McDonalds & NYSE & MCD & Fast food \\
\hline
\end{tabular}


Fičura, M.: Forecasting Cross-Section of Stock Returns with Realized Moments.

\begin{tabular}{llll} 
Merck & NYSE & MRK & Pharmaceuticals \\
Microsoft & NASDAQ & MSFT & Software \\
Navistar & NYSE & NAV & Automotive \\
Nike & NYSE & NKE & Apparel \\
Owens-Illinois & NYSE & OI & Glass \\
Pfizer & NYSE & PFE & Pharmaceuticals \\
Primerica & NYSE & PRI & Financial services \\
Procter \& Gamble & NYSE & PG & Consumer goods \\
Travelers & NYSE & TRV & Insurance \\
UnitedHealth Group & NYSE & UNH & Managed health care \\
United States Steel & NYSE & X & Steel \\
United Technologies & NYSE & UTX & Conglomerate \\
Vale & NYSE & VALE & Metals \\
Verizon & NYSE & VZ & Telecommunication \\
Visa & NYSE & V & Consumer banking \\
Wal-Mart & NYSE & WMT & Retail \\
Walgreens Boots Alliance & NASDAQ & WBA & Retail \\
Walt Disney & NYSE & DIS & Broadcasting andentertainment \\
\hline Source: Prepared & &
\end{tabular}

Source: Prepared by the author by using the historical data about DJIA components from: https://us.spindices.com/indexology/djia-and-sp-500/the-changing-djia 